\title{
High Frequency Coupling Coefficient between the Coplanar Resonator and Radio Frequency SQUID
}

\author{
H. R. Yi, Y. Zhang, H. Bousack, and A. I. Braginski \\ Institut für Schicht- und Ionentechnik (ISI), Forschungszentrum Jülich (FZJ), D-52425 Jülich, Germany
}

\begin{abstract}
The coupling coefficient $\left(\mathrm{k}^{2}\right)$ between the superconducting coplanar resonator and the flip chip coupled washer radio frequency SQUID was calculated using computer simulation. The coplanar resonator is formed by two coplanar lines surrounding a flux concentrator with each line having a slit. For a $3 \mathrm{~mm} \times 3 \mathrm{~mm}$ washer SQUID with a $100 \mu \mathrm{m} \times 100$ $\mu \mathrm{m}$ loop coupled to a coplanar resonator with the slits on opposite sides, we estimated $k^{2}=7.1 \times 10^{-3}$. However, if the same SQUID is coupled to a coplanar resonator with parallel slits and with a short circuit at the $90^{\circ}$ position with respect to the opening slit, $k^{2}$ of only $0.5 \times 10^{-3}$ is obtained, which indicates that the $k^{2}$ is strongly dependent on the resonator layout. The simulation also shows that $\mathrm{k}^{2}$ is dependent on the SQUID washer size and the separation distance between the washer SQUID and the coplanar resonator.
\end{abstract}

\section{INTRODUCTION}

A tank circuit (resonator) is used for the operation of a radio frequency superconducting quantum interference device (rf SQUID). Normally, the rf SQUID is inductively coupled to the inductor of the resonator. For optimum operation of rf SQUIDs, the resonator should have a high unloaded quality factor $Q_{0}$, a high resonant frequency $f_{0}$ (with $\mathrm{f}_{0}<\mathrm{R}_{\mathrm{n}} / 2 \pi \mathrm{L}_{\mathrm{s}}$, where $\mathrm{R}_{\mathrm{n}}$ is the normal resistance of the junction, and $\mathrm{L}_{\mathrm{s}}$ the SQUID inductance), and an appropriate coupling coefficient $\left(k^{2}=M^{2} / L_{1} L_{s}\right)$ between the resonator and the SQUID that satisfies $k^{2} Q_{L} \geq 1[1-2]$. Here, $L_{1}$ is the coupling inductance of the resonator, $M$ is the mutual inductance between the $\mathrm{L}_{1}$ and $\mathrm{L}_{5}$, and $\mathrm{Q}_{\mathrm{L}}$ is the loaded quality factor of the resonator.

The $\mathrm{Q}_{0}$ and $\mathrm{f}_{0}$ can be measured with the help of a network analyzer. However, the direct measurement of $k^{2}$ is very difficult for planar SQUIDs. Since $\mathrm{k}^{2}$ is a very important parameter for optimizing the design of rf SQUIDs, in this work we have used simulation to estimate $\mathrm{k}^{2}$ between the superconducting coplanar resonator and the rf SQUID.

\section{COPLANAR RESONATOR}

Recently, the concept of using a superconducting coplanar resonator as a tank circuit for rf SQUID was

Manuscript received September 14, 1998.

H. R. Yi, present address: Institut für Festkörperforschung (IFF), FZJ, 52425 Jülich, Germany; E-mail: h.yi@fz-juelich.de introduced [3-4]. The best white flux noise for a SQUID of inductance $260 \mathrm{pH}$ measured with the superconducting coplanar resonator was $8.5 \mu \Phi_{0} / \mathrm{Hz}^{1 / 2}$ at $77 \mathrm{~K}$ in a magnetic shield [4]. Here, $\Phi_{0}$ is the flux quantum. This noise level was considerably lower than the best white flux noise level $(20$ $\mu \Phi_{0} / \mathrm{Hz}^{1 / 2}$ ) for the same SQUID measured with an improved traditional tank circuit [5].

Fig. 1 shows the schematic layout of a superconducting coplanar resonator [4]. It is formed by two coplanar lines surrounding a flux concentrator with each line having a slit. The resonator is patterned from a $200 \mathrm{~nm}$-thick c-axis oriented epitaxial $\mathrm{YBa}_{2} \mathrm{Cu}_{3} \mathrm{O}_{7}$ (YBCO) film grown on $1 \mathrm{~mm}$ thick (001) $\mathrm{LaAlO}_{3}$ (LAO) substrate. Besides the substrate size, its resonant frequency also depends on the mutual angular position $(\theta)$ of the two microstrip slits, as well as on the presence or absence of a short circuit between the two microstrip lines. Unloaded quality factors as high as $2 \times 10^{4}$ can be obtained [4]. A washer rf SQUID is coupled to the central hole of the superconducting flux concentrator by the flip-chip configuration, thus forming a magnetometer.

The superconducting flux concentrator couples both $\mathrm{dc}$ (low frequency) and rf signals to the rf SQUID. Its inductance can be calculated by an empirical formula [6]:

$L_{1}=\frac{1.86}{\pi} \mu_{0}\left(\frac{D+d}{2}\right)\left[\ln \frac{D+d}{D-d}+0.42\right]$,

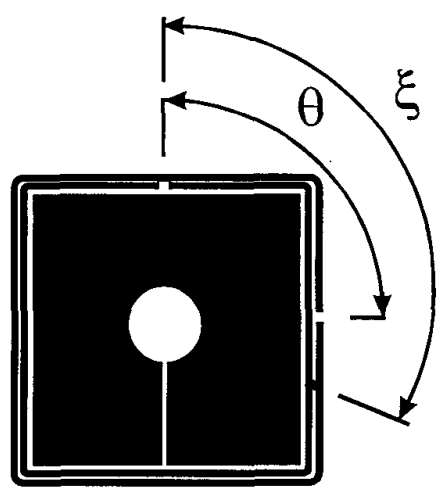

Fig. 1. Schematic of a coplanar resonator integrated with a flux concentrator. $\theta$ denotes the mutual angular position of the coplanar resonator openings. $\xi$ denotes the angular position of the short circuit between the two striplines with respect to the opening of the inner stripline. A washer rf SQUID is placed onto the concentrator to form a flip chip package [4] 
where $\mathrm{D}$ and $\mathrm{d}$ are the outer and inner dimensions of the square loop (flux concentrator), and $\mu_{0}$ is the vacuum permeability. When an external dc magnetic field is applied, the superconducting flux concentrator compresses the magnetic flux to its central hole, thus increasing the effective area of the magnetometer.

Under the linear approximation (i.e. neglecting the phase shift across the junction, except when a transition occurs), the total flux threading the SQUID ring $\left(\Phi_{i}\right)$ is related to an external flux $\Phi_{\mathrm{x}}$ by [1]:

$$
\Phi_{i}=\Phi_{x}+L_{s} i_{s}=n \Phi_{0},\left(\mathrm{i}_{\mathrm{s}} \leq \mathrm{i}_{\mathrm{c}}, \mathrm{n}=0, \pm 1, \pm 2, \ldots\right)
$$

where $i_{s}$ is the supercurrent flowing in the SQUID ring and $i_{c}$ the critical current of the junction. Consider a coplanar resonator driven by an $\mathrm{rf}$ current source at the resonant frequency $f_{0}$. If the mutual inductance between $L_{s}$ and $L_{1}$ is $M$ and current flowing in $L_{1}$ is $i_{1}$ (effective value), $\Phi_{x}=\mathrm{Mi}_{1}$, then as long as the internal flux state remains the same, i.e. $\mathrm{n}=0$ in (2), there will be a simple linear relationship between $i_{1}$ and $i_{s}[1]$, and $M$ can be expressed as:

$$
M=-L_{s} i_{s} / i_{1}
$$

Put (3) to the definition of $\mathrm{k}^{2}$, we have:

$$
k^{2}=\frac{M^{2}}{L_{1} L_{s}}=\frac{L_{s}}{L_{1}}\left(\frac{i_{s}}{i_{1}}\right)^{2}
$$

$\mathrm{L}_{\mathrm{s}}$ can be calculated by empirical formulae. For a SQUID having a square hole, $L_{s}$ is given by [7]:

$$
L_{s}=1.25 \mu_{0} a
$$

where $a$ is the lateral length of the square hole. One can estimate $\mathrm{k}^{2}$, once the ratio $\mathrm{i}_{\mathrm{s}} / \mathrm{i}_{1}$ is known. Below, we use em software to simulate $\mathrm{i}_{\mathrm{s}} / \mathrm{i}_{1}$.

\section{SiMULATION DETAILS}

The em software, developed by Sonnet Software, Inc. [8], is an electromagnetic analysis, which calculates S-parameters for arbitrary three-dimensional planar passive circuits. In a recent work [9], we have used the em software to simulate superconducting coplanar resonators. The resonant frequencies obtained in the simulation were in good agreement with the experimentally measured values for the different geometrical layouts.

A two-port system similar to the experimental one has been constructed for the simulation. Two copper loops with outer dimension of $3 \mathrm{~mm} \times 3 \mathrm{~mm}$ and a line width of $0.5 \mathrm{~mm}$ are used as the input and output loops, respectively. For each loop, one end is connected to a $50 \Omega$ coaxial transmission cable (i.e. port) and another end is connected to the ground. An input rf voltage of $50 \mu \mathrm{V}$ was used for the simulation. This voltage value, which determines the values of $i_{1, r f}$ and $\mathrm{i}_{\mathrm{s}, \mathrm{f}}$, is in the voltage range used in the experiments.

The central part is the coplanar resonator patterned from a superconducting YBCO film deposited on a LAO single crystal substrate, and a washer rf SQUID coupled to the central hole of the superconducting flux concentrator by the flip-chip configuration. The separation between the $\mathrm{rf}$ SQUID and the concentrator is $h_{s}$ and the separation media is air.

The lateral dimension of the square washer SQUID is $d_{s}$. The SQUID hole is $100 \mu \mathrm{m} \times 100 \mu \mathrm{m}$ and the junction width is $50 \mu \mathrm{m}$. The dimension of the concentrator washer is 8.5 $\mathrm{mm} \times 8.5 \mathrm{~mm}$. The inner hole of the concentrator is $1.5 \mathrm{~mm}$ $\times 1.5 \mathrm{~mm}$. The width of the two microstrip lines is $100 \mu \mathrm{m}$. The width of the short circuit, the width of the slits, the distance between the concentrator and the inner microstrip line, and between the microstrip lines themselves were all 50 $\mu \mathrm{m}$. The LAO substrate is $10 \mathrm{~mm} \times 10 \mathrm{~mm}$ large and $1 \mathrm{~mm}$ thick. It has a loss tangent of $4 \times 10^{-5}$ at around $800 \mathrm{MHz}$ [9], and a dielectric constant $\varepsilon_{\mathrm{r}}=24$ at $77 \mathrm{~K}$ [10]. The input loop is placed $6 \mathrm{~mm}$ above the coplanar resonator, and the output loop is placed $6 \mathrm{~mm}$ below the dielectric substrate.

The circuit was simulated at different frequencies. The resonant frequency $\left(f_{0}\right)$ is determined as the frequency that corresponds to the maximum value of the transmitted signals. Then the current distribution is simulated at the resonant frequency. From the current distributions on the superconducting coplanar resonator and washer SQUID, we determine $i_{5}$ and $i_{1}$.

\section{RESULTS AND DISCUSSION}

We simulated the coplanar resonator of $\theta=180^{\circ}$ loaded with a square washer SQUID of $d_{s}=3 \mathrm{~mm}$ and the hole size of $100 \mu \mathrm{m} \times 100 \mu \mathrm{m}$. The SQUID is loaded to the resonator by the flip chip configuration with a separation distance $h_{s}=5$ $\mu \mathrm{m}$. Fig. 2 shows the simulated current distribution at the resonant frequency (i.e. working frequency) $f_{0}=839 \mathrm{MHz}$. The upper part shows the current distribution on the coplanar resonator and the lower part shows the current distribution on the washer SQUID. For the YBCO film, the light area represents a high current density and the dark area represents a low current density. For each strip line, the highest current density appears in the center part, while no current is present at the two ends, i.e. the open slit. This current distribution is similar to a half-wavelength resonator.

The $i_{\mathrm{s}}$ was determined as the current passing through the junction. The $i_{1}$ was determined at the $90^{\circ}$ position with respect to the opening slit on the superconducting flux concentrator. Actually, we used the Autoscale function of the emvu [8] program to get the $\mathrm{i}_{1, \mathrm{ff}}$ value for this coplanar resonator layout. From Fig. 2 we obtained $i_{1}=5.12 \mu \mathrm{A}$, and $\mathrm{i}_{\mathrm{s}}=2.10 \mu \mathrm{A}$. Using (1) and $\mathrm{D}=8.5 \mathrm{~mm}, \mathrm{~d}=1.5 \mathrm{~mm}$, we 
TABLE I
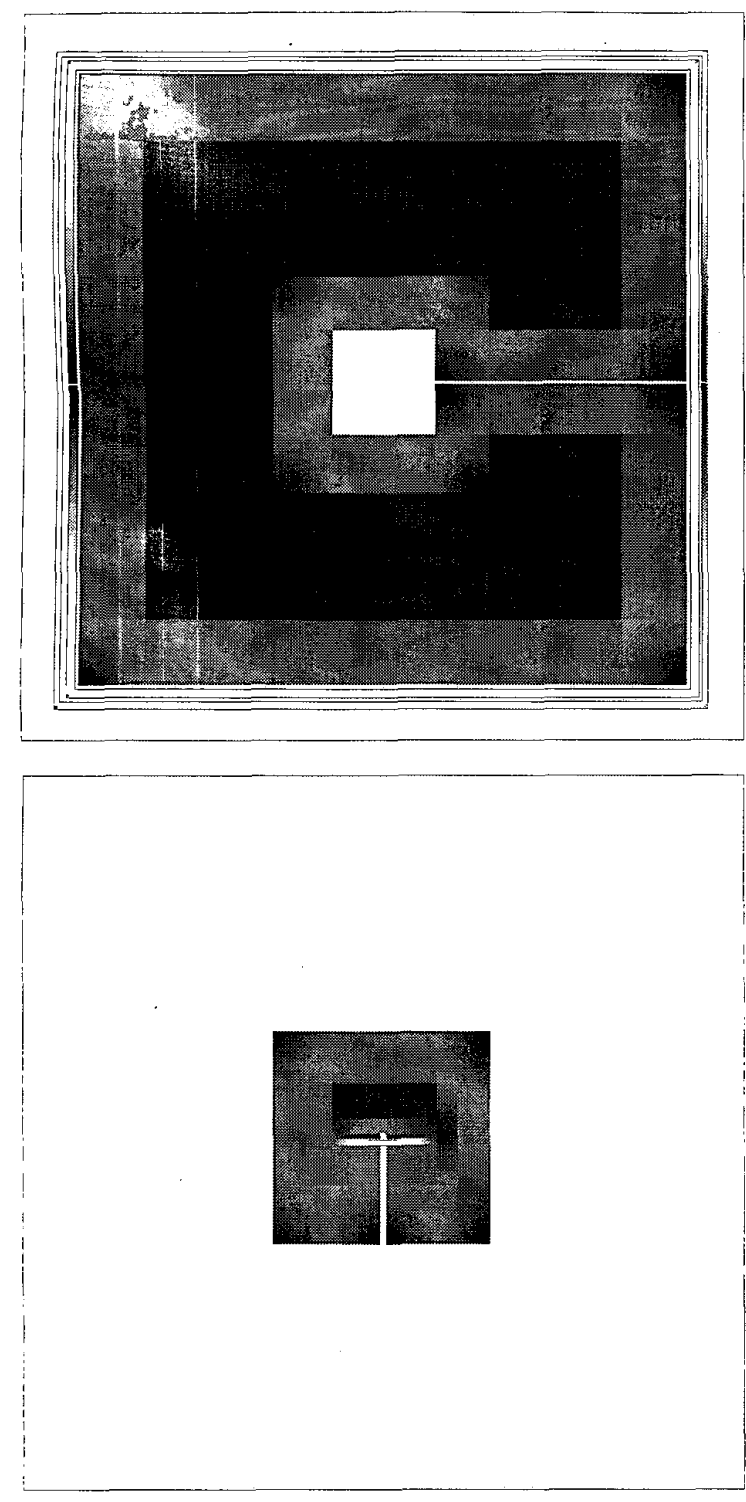

Fig. 2. Simulated current distribution on the coplanar resonator of $\theta=180^{\circ}$ (upper part) and on the loaded square washer SQUID by the flip chip configuration (lower part) at the resonant frequency $\mathrm{f}_{0}=839 \mathrm{MHz}$. The current scale is from 0 to $1.5 \times 10^{-2} \mathrm{~A} / \mathrm{m}$ with the light area representing high current density and the dark area representing low current density. The SQUID washer dimension is $3 \mathrm{~mm} \times 3 \mathrm{~mm}$ and the SQUID hole size is 100 $\mu \mathrm{m} \times 100 \mu \mathrm{m}$. The flux concentrator dimension is $8.5 \mathrm{~mm} \times 8.5 \mathrm{~mm}$ and the inner hole size is $1.5 \mathrm{~mm} \times 1.5 \mathrm{~mm}$.

calculated $\mathrm{L}_{1}=3.57 \mathrm{nH}$. Using (5) and $a=100 \mu \mathrm{m}$, we calculated $L_{s}=0.15 \mathrm{nH}$. Put these values to (4), we obtained $\mathrm{k}^{2}=7.1 \times 10^{-3}$.

The experimental loaded quality factor $\mathrm{Q}_{\mathrm{L}}$ of the superconducting coplanar resonator, as measured by Zeng
DEPENDENCE OF THE SIMULATED $i_{s}$ AND $i_{1}$, AND THE CALCULATED $k^{2}$ ON THE PARAMETERS OF $d_{s}, h_{s}$, AND $R_{\mathrm{J}}$. SYMBOLS ARE DEFINED IN THE TEXT.

\begin{tabular}{lllll}
\hline $\mathrm{d}_{\mathrm{s}}(\mathrm{mm})$ & $\mathrm{h}_{\mathrm{s}}(\mu \mathrm{m})$ & $\mathrm{i}_{\mathrm{s}}(\mu \mathrm{A})$ & $\mathbf{i}_{1}(\mu \mathrm{A})$ & $\mathrm{k}^{2}\left(10^{-3}\right)$ \\
\hline 2 & 5 & 2.13 & 5.38 & 6.6 \\
2.5 & 5 & 1.93 & 4.85 & 6.7 \\
3 & 5 & 2.10 & 5.12 & 7.1 \\
3.5 & 5 & 2.13 & 4.75 & 8.4 \\
4 & 5 & 2.25 & 4.48 & 10.6 \\
5 & 5 & 2.50 & 4.90 & 10.9 \\
3 & 10 & 2.03 & 5.12 & 6.6 \\
3 & 20 & 1.99 & 5.18 & 6.2 \\
3 & 30 & 1.97 & 5.23 & 6.0 \\
3 & 40 & 1.95 & 5.28 & 5.7 \\
3 & 50 & 1.93 & 5.32 & 5.5 \\
3 & 60 & 1.91 & 5.36 & 5.3 \\
3 & 100 & 1.83 & 5.46 & 4.7 \\
3 & 150 & 1.73 & 5.51 & 4.1 \\
3 & 200 & 1.63 & 5.51 & 3.7 \\
\hline
\end{tabular}

[11], was about 300 to 400 . With this $\mathrm{Q}_{\mathrm{L}}$ and the calculated $\mathrm{k}^{2}$, the condition for optimum operation of $\mathrm{rf}$ SQUID, i.e. $k^{2} Q_{L} \geq 1$, is satisfied.

In practice, rf SQUIDs of different washer size are used. Also, in the flip chip configuration, the separation distance between the SQUID and resonator may differ from time to time. We also simulated the same coplanar resonator coupled by rf SQUIDs of different washer sizes $\left(d_{s}\right)$ and at different separation distances $\left(h_{s}\right)$. The simulated $i_{1}$ and $i_{s}$ and the calculated $k^{2}$ are shown in Table I. For a fixed $h_{s}=5 \mu \mathrm{m}, k^{2}$ increases with increasing the washer size. For a fixed washer size of $d_{s}=3 \mathrm{~mm}, \mathrm{k}^{2}$ decreases with increasing the separation

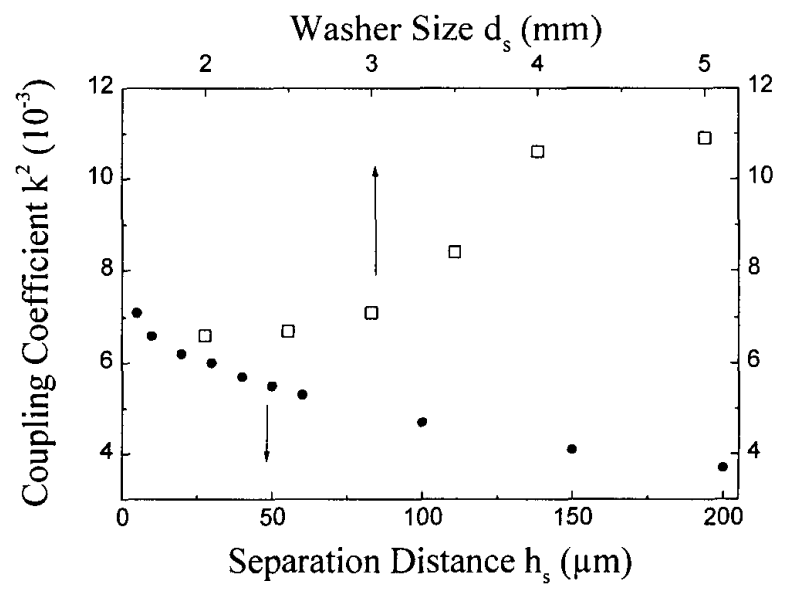

Fig. 3. Dependence of the coupling coefficient $\mathrm{k}^{2}$ on the washer size (open squares) and the separation distance (filled circles). 


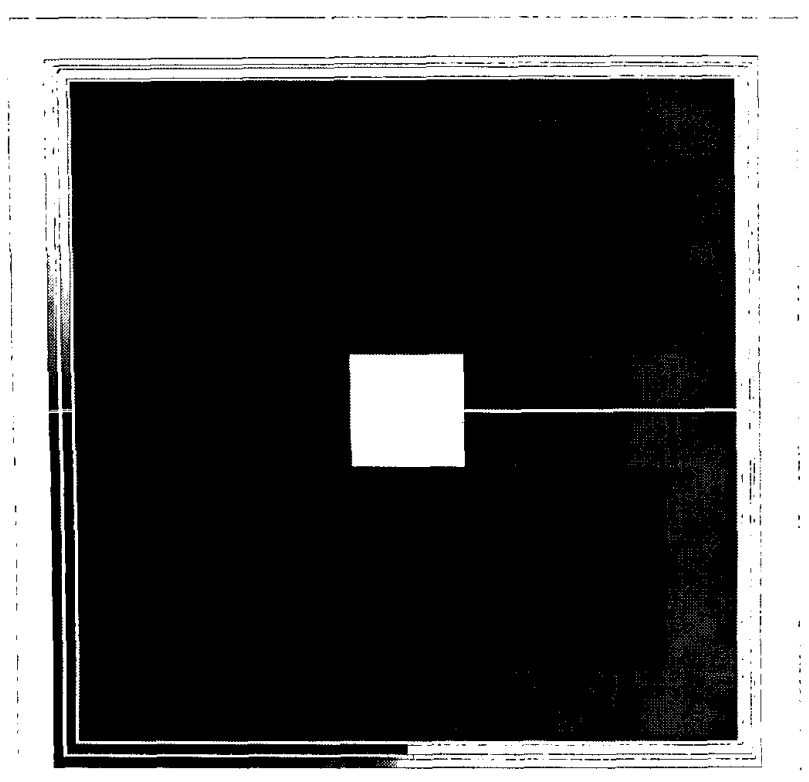

Fig. 4. Simulated current distribution. on the coplanar resonator with the angular position $\theta=0^{\circ}$ and with a short circuit at $\xi=90^{\circ}$ loaded by a $3 \mathrm{~mm} \times$ $3 \mathrm{~mm}$ washer SQUID with the hole size of $100 \mu \mathrm{m} \times 100 \mu \mathrm{m}$ at the resonant frequency $f_{0}=808 \mathrm{MHz}$. The current scale is from 0 to $7.5 \times 10^{-1}$ $\mathrm{A} / \mathrm{m}$.

distance. The dependence of $k^{2}$ on $d_{s}$ at a fixed $h_{s}=5 \mu \mathrm{m}$, and on $h_{s}$ for a fixed $d_{s}=3 \mathrm{~mm}$ is plotted in Fig. 3 .

Fig. 4 shows the current distribution for another superconducting coplanar resonator layout with the angular position $\theta=0^{\circ}$ and with a short circuit at $\xi=90^{\circ}$ loaded with the same washer SQUID as in Fig. 2. The separation distance was also chosen to be $h_{s}=5 \mu \mathrm{m}$. In this case, $i_{1}=0.73 \mu \mathrm{A}$ and $\mathrm{i}_{\mathrm{s}}=0.08 \mu \mathrm{A}$ are obtained, resulting in a coupling coefficient of $\mathrm{k}^{2}=0.5 \times 10^{-3}$. This value of the coupling coefficient is much lower than that of the coplanar resonator layout shown in Fig. 2. Note that in this case, the $i_{1}$ and $i_{s}$ are also lower than that of the resonator layout of Fig. 2 for the same input voltage (i.e. $50 \mu \mathrm{V}$ ).

With this $\mathrm{k}^{2}=0.5 \times 10^{-3}$ and the experimental loaded quality factor $Q_{L} \approx 400$, the condition for optimum operation of $\mathrm{rf}$ SQUID, $k^{2} Q_{L} \geq 1$, is not satisfied. Thus, this resonator layout is not a good choice for $\mathrm{rf}$ SQUIDs. This conclusion is consistent with the experimental result for this coplanar resonator layout [12].

\section{SUMMARY}

The electromagnetic analysis program em has been used to simulate the superconducting coplanar resonator loaded with a washer rf SQUID by the flip chip configuration. With the simulated current distribution on the superconducting coplanar resonator and the loaded rf SQUID at the resonant frequency, we were able to estimate the coupling coefficient $\mathrm{k}^{2}$ between the superconducting coplanar resonator and $\mathrm{rf}$ SQUID. For a coplanar resonator with the angular position $\theta=0^{\circ}$, loaded by a $3 \mathrm{~mm} \times 3 \mathrm{~mm}$ washer SQUID with a 100 $\mu \mathrm{m} \times 100 \mu \mathrm{m}$ loop, $\mathrm{k}^{2}$ is estimated to be $7.1 \times 10^{-3}$. This $\mathrm{k}^{2}$ value satisfies the condition of optimum operation of $\mathrm{rf}$ SQUID: $k^{2} Q_{L} \geq 1$ for the measured loaded quality factor $Q_{L}$. However, when the same SQUID is coupled to the superconducting coplanar resonator with the angular position $\theta=0^{\circ}$ and with a short circuit at $\xi=90^{\circ}, \mathrm{k}^{2}$ of only $0.5 \times 10^{-3}$ is obtained. This $k^{2}$ value is too small to satisfy $k^{2} Q_{L} \geq 1$. This indicates that $\mathrm{k}^{2}$ is strongly dependent on the resonator layout. The simulation also shows that $k^{2}$ increases with increasing washer size and decreases with increasing separation distance between the SQUID and the coplanar resonator.

\section{ACKNOWLEDGMENT}

We would like to thank H. J. Krause and M. Grüneklee for their readings and corrections of the manuscript. HRY also thanks F. Schmidt and E. Sodtke for their kind help in the computer programming. Helpful discussions with $\mathrm{N}$. Klein and X. H. Zeng are gratefully acknowledged.

\section{REFERENCES}

[1] J. C. Gallop, SQUIDs, the Josephson Effects and Superconducting Electronics. Adam Hilger, Bristol, 1991.

[2] M. Mück, Developments and Characterization of low noise rf SQUIDs. Habilitationsschrift, Gießen: University of Gießen, 1995.

[3] Y. Zhang, W. Zander, J. Schubert, F. Rüders, H. Soltner, M. Banzet, N. Wolters, X. H. Zeng, and A. I. Braginski, "Operation of highsensitivity radio frequency superconducting quantum interference device magnetometers with superconducting coplanar resonators at 77 K," Appl. Phys. Lett., vol. 71, pp. 704-706, August 1997.

[4] Y. Zhang, N. Wolters, X. H. Zeng, J. Schubert, W. Zander, H. Soltner, H. R. Yi, M. Banzet, F. Rüders, and A. I. Braginski, "Washer rf SQUID magnetometers with coplanar resonators at $77 \mathrm{~K}$," Applied Superconductivity, in press.

[5] D. F. He, X. H. Zeng, H. J. Krause. H. Soltner, F. Rüders, and Y Zhang, "Radio frequency SQUIDs operating at $77 \mathrm{~K}$ with $1 \mathrm{GHz}$ lumped-element tank circuits," Appl. Phys. Lett., vol. 72, pp. 969 971, February 1998.

[6] F. Ludwig, E. Dantsker, D. Koelle, R. Kleiner, A. H. Miklich, and J Clarke, "Multilayer magnetometers based on high- $T_{c}$ SQUIDs," Applied Superconductivity, Vol. 3, pp. 383-398, July-October 1995.

[7] W. H. Chang, "Numerical calculation of inductances of a multisuperconductor transmission line system," IEEE Trans. Mag., vol. 17, pp. 764-766, 1981.

[8] Sonnet Software, Inc. Liverpool, NY.

[9] H. R. Yi, Y. Zhang, and A. I. Braginski, "Numerical modeling of superconducting coplanar resonators for radio frequency SQUIDs," Appl. Phys. Lett., in press.

[10] G. A. Samara. "Low-temperature dielectric properties of candidate substrates for high-temperature superconductors: $\mathrm{LaAlO}_{3}$ and $\mathrm{ZrO}_{2}: 9.5 \mathrm{~mol} \% \mathrm{Y}_{2} \mathrm{O}_{3}, " J$. Appl. Phys., vol. 68, pp. 4214-4219, October 1990.

[11] X. H. Zeng, unpublished.

[12] Y. Zhang, unpublished. 